\title{
On the quasi-weak drop property
}

by

\author{
J. H. QIU (Suzhou)
}

\begin{abstract}
A new drop property, the quasi-weak drop property, is introduced. Using streaming sequences introduced by Rolewicz, a characterisation of the quasi-weak drop property is given for closed bounded convex sets in a Fréchet space. From this, it is shown that the quasi-weak drop property is equivalent to weak compactness. Thus a Fréchet space is reflexive if and only if every closed bounded convex set in the space has the quasi-weak drop property.
\end{abstract}

1. Introduction. Let $(X,\|\|)$ be a Banach space and let $B(X):=$ $\{x \in X:\|x\| \leq 1\}$ be its closed unit ball. By a drop $D\left(x_{0}, B(X)\right)$ determined by a point $x_{0} \in X \backslash B(X)$, we mean the convex hull of the set $\left\{x_{0}\right\} \cup B(X)$. Daneš [1] proved that for every closed set $A$ at positive distance from the $B(X)$, there exists an $x_{0} \in A$ such that $D\left(x_{0}, B(X)\right) \cap A=\left\{x_{0}\right\}$. Rolewicz [11], modifying the Daneš drop theorem assumption, introduced the following definition: the norm \|\| has the drop property if for every closed set $A$ disjoint from $B(X)$ there exists an $x_{0} \in A$ such that $D\left(x_{0}, B(X)\right) \cap A=\left\{x_{0}\right\}$. He also proved that if the norm \|\| has the drop property then $(X,\|\|)$ is reflexive (see [11, Theorem 5]).

Giles, Sims and Yorke [3] defined the following variant: the norm || || has the weak drop property if for every weakly sequentially closed set $A$ disjoint from $B(X)$, there exists an $x_{0} \in A$ such that $D\left(x_{0}, B(X)\right) \cap A=\left\{x_{0}\right\}$, and they showed that this property is equivalent to $(X,\|\|)$ being reflexive. Instead of drops formed from the closed unit ball Kutzarova [7] considered drops formed from any closed bounded convex set $B$, and defined that $B$ has the drop property if for any closed set $A$ disjoint from $B$ there exists an $x_{0} \in A$ such that $D\left(x_{0}, B\right) \cap A=\left\{x_{0}\right\}$. Moreover, according to Giles and Kutzarova [2], a closed bounded convex set $B$ has the weak drop property if for every weakly sequentially closed set $A$ disjoint from $B$ there exists an $x_{0} \in A$ such that $D\left(x_{0}, B\right) \cap A=\left\{x_{0}\right\}$.

2000 Mathematics Subject Classification: 46A55, 46B20.

Key words and phrases: Fréchet space, reflexive space, quasi-weak drop property, weakly compact set. 
Concerning the weak drop property of closed bounded convex sets, the following significant characterisation is known.

Theorem 1.1 (see [2, Theorem 3] or [8, Proposition 4.4.7]). For a closed bounded convex set with $0 \in B$ in a Banach space $(X,\|\|)$, the following statements are equivalent:

(i) $B$ has the weak drop property;

(ii) $B$ is weakly compact.

In this paper, by substituting "weakly closed set $A$ " for "weakly sequentially closed set $A$ " in the definition of the weak drop property introduced by Giles and Kutzarova, we define a new drop property - the quasi-weak drop property, which seems weaker than the weak drop property for closed bounded convex sets in locally convex spaces. Using streaming sequences introduced by Rolewicz [11], we prove that a closed bounded convex set $B$ in a Fréchet space (i.e. a complete metrizable locally convex space) has the quasi-weak drop property if and only if every stream in $X \backslash B$ has a weak cluster point. From this we deduce that the quasi-weak drop property is equivalent to weak compactness and hence it is equivalent to the weak drop property for closed bounded convex sets in a Fréchet space. Thus a Fréchet space is reflexive if and only if every closed bounded convex subset has the quasi-weak drop property.

\section{Quasi-weak drop property}

Definition 2.1. Let $B$ be a closed bounded convex set in a locally convex space $X$. If for any weakly closed set $A$ disjoint from $B$, there exists an $x_{0} \in A$ such that $D\left(x_{0}, B\right) \cap A=\left\{x_{0}\right\}$, then $B$ is said to have the quasi-weak drop property. The norm \|\| of a Banach space $(X,\|\|)$ is said to have the quasi-weak drop property if the closed unit ball $B(X)$ has this property.

Associated with the drop property Rolewicz introduced a useful sequential concept. Given a closed bounded convex set $B$, a sequence $\left\{x_{n}\right\}$ in $X \backslash B$ such that $x_{n+1} \in D\left(x_{n}, B\right)$ for all $n \in \mathbb{N}$ is called a stream. Rolewicz [11, Proposition 2] proved that the norm \|\| has the drop property if and only if each stream in $X \backslash B(X)$ contains a convergent subsequence, and this was generalized in [3, Theorem 5] to the weak form that the norm \|\| has the weak drop property if and only if each stream in $X \backslash B(X)$ contains a weakly convergent subsequence. Moreover, Giles and Kutzarova [2, Theorem 2] proved that a closed bounded convex set $B$ in a Banach space $(X,\|\|)$ has the drop (resp. weak drop) property if and only if every stream in $X \backslash B$ has a norm (resp. weak) convergent subsequence. Inspired by the above results, we give a characterisation of the quasi-weak drop property for closed bounded convex sets as follows. 
Theorem 2.1. A closed bounded convex set $B$ in a Fréchet space $(X, d)$ has the quasi-weak drop property if and only if every stream in $X \backslash B$ has a weak cluster point.

In order to prove Theorem 2.1 we need the following lemmas.

LEMma 2.1. Let $\left\{x_{n}\right\}$ be a sequence in a Hausdorff topological space $X$. If $\left\{x_{n}\right\}$ has no cluster point, then the set $C:=\left\{x_{n}: n \in \mathbb{N}\right\}$ is closed.

Lemma 2.1 is classic and well known, so we omit its proof. We call an absolutely convex bounded set $G$ in a locally convex space a Banach disk if $E_{G}:=\left(\operatorname{sp}[G], p_{G}\right)$ is a Banach space, where $\operatorname{sp}[G]$ denotes the vector subspace spaned by $G$ and $p_{G}$ denotes the Minkowski gauge of $G$ (see, for example, [6, II, p. 70] or [9, Definition 3.2.4]). It is easy to see that every closed absolutely convex bounded set in a Fréchet space is a Banach disk. Now we can give the following:

Lemma 2.2. Let $B$ be a closed bounded convex set in a Fréchet space $(X, d)$ and $A$ be a weakly closed set disjoint from $B$. If for any $x \in A$, $D(x, B) \cap A \neq\{x\}$, then for any $z \in A, d(D(z, B) \cap A, B)=0$. Here $d(E, F)$ denotes $\inf \{d(a, b): a \in E, b \in F\}$ and $d(a, b)$ denotes the distance between $a$ and $b$.

Proof. Assume that there exists a $z_{0} \in A$ such that $d\left(D\left(z_{0}, B\right) \cap A, B\right)$ $>0$. Then $0 \notin \operatorname{cl}\left(D\left(z_{0}, B\right) \cap A-B\right)$. Hence there exists an absolutely convex 0-neighbourhood $V$ such that

$$
\left(D\left(z_{0}, B\right) \cap A-B\right) \cap V=\emptyset .
$$

Denote the closed absolutely convex hull of $B \cup\left\{z_{0}\right\}$ in $(X, d)$ by $G$. Then $G$ is a Banach disk, i.e., $E_{G}=\left(\operatorname{sp}[G], p_{G}\right)$ is a Banach space. Clearly $D\left(z_{0}, B\right) \cap A$ is a closed set and $B$ is a closed bounded convex set in the Banach space $E_{G}$. Since $G$ is bounded in $(X, d)$, there exists $\lambda>0$ such that $\lambda G \subset V$. Thus by (1) we have

$$
\left(D\left(z_{0}, B\right) \cap A-B\right) \cap(\lambda G)=\emptyset,
$$

which leads to $p_{G}(a-b) \geq \lambda$ for all $a \in D\left(z_{0}, B\right) \cap A$ and $b \in B$. Now in the Banach space $E_{G}$, the distance between $D\left(z_{0}, B\right) \cap A$ and $B$ is positive. Using the Daneš Theorem, we conclude that there exists $x_{0} \in D\left(z_{0}, B\right) \cap A$ such that

$$
D\left(x_{0}, B\right) \cap D\left(z_{0}, B\right) \cap A=\left\{x_{0}\right\} .
$$

From $x_{0} \in D\left(z_{0}, B\right)$, we see that $D\left(x_{0}, B\right) \subset D\left(z_{0}, B\right)$, so by (2) we have

$$
D\left(x_{0}, B\right) \cap A=\left\{x_{0}\right\} .
$$

This contradicts the assumption that $D(x, B) \cap A \neq\{x\}$ for every $x \in A$. 
Proof of Theorem 2.1. (i) Suppose that there exists a stream $\left\{x_{n}\right\}$ in $X \backslash B$ which has no weak cluster point. By Lemma 2.1, the set $A:=\left\{x_{n}\right.$ : $n \in \mathbb{N}\}$ is weakly closed. We may assume that $x_{n} \neq x_{m}$ for any $n \neq m$. Note that $x_{n+1} \in A, x_{n+1} \in D\left(x_{n}, B\right)$ and $x_{n+1} \neq x_{n}$ for all $n \in \mathbb{N}$, hence there is no $n \in \mathbb{N}$ such that $D\left(x_{n}, B\right) \cap A=\left\{x_{n}\right\}$. This means that $B$ does not have the quasi-weak drop property.

(ii) Conversely, suppose that every stream in $X \backslash B$ has a weak cluster point, but $B$ does not have the quasi-weak drop property, that is, there exists a weakly closed set $A$ disjoint from $B$ such that for any $x \in A, D(x, B) \cap A$ $\neq\{x\}$. By Lemma 2.2, for any $z \in A, d(D(z, B) \cap A, B)=0$. Taking any fixed $x_{1} \in A$ and any fixed $y_{1} \in B$, we have

$$
d\left(D\left(x_{1}, B\right) \cap A, B\right)=0 .
$$

Thus there exist $x_{2} \in D\left(x_{1}, B\right) \cap A$ and $y_{2} \in B$ such that $d\left(x_{2}, y_{2}\right)<1 / 2$. Since

$$
d\left(D\left(x_{2}, B\right) \cap A, B\right)=0,
$$

there exist $x_{3} \in D\left(x_{2}, B\right) \cap A$ and $y_{3} \in B$ such that $d\left(x_{3}, y_{3}\right)<1 / 3$. Continuing this process we obtain sequences $\left\{x_{n}\right\} \subset A \subset X \backslash B$ and $\left\{y_{n}\right\} \subset B$. Clearly $\left\{x_{n}\right\}$ is a stream in $X \backslash B$. By our assumption, $\left\{x_{n}\right\}$ has a weak cluster point $x_{0}$. Since $A$ is weakly closed and $\left\{x_{n}\right\} \subset A$, we have $x_{0} \in A$. On the other hand,

$$
d\left(x_{n}, y_{n}\right)<1 / n \rightarrow 0 \quad \text { as } n \rightarrow \infty .
$$

Hence $y_{n}-x_{n} \stackrel{n}{\rightarrow} 0$ in $(X, d)$ and so in $\left(X, \sigma\left(X, X^{*}\right)\right)$. Thus $x_{0}$ is also a weak cluster point of the sequence $\left\{y_{n}\right\} \subset B$. This shows that $x_{0} \in B$ since the closed convex set $B$ is also weakly closed. Therefore $x_{0} \in B \cap A$, which contradicts the assumption that $A$ is disjoint from $B$.

In order to investigate the relationship between the drop property, weak drop property and quasi-weak drop property, we need to recall some facts concerning closed sets, weakly sequentially closed sets and weakly closed sets (see, for example, [4], [6] or [12]). Let $(X, d)$ be a metrizable locally convex space and let $X^{*}$ be its topological dual. Clearly every weakly closed set in $X$ is weakly sequentially closed and every weakly sequentially closed set is closed in $(X, d)$. However, a closed set in $(X, d)$ need not be weakly sequentially closed (see Example 2.1 below) and a weakly sequentially closed set need not be weakly closed (see Example 2.2).

EXAMPLE 2.1. Let $c_{0}$ be the space of all scalar sequences convergent to 0 with the supremum norm. Then $\left(c_{0},\|\|\right)^{*}=l^{1}$. Set $S=\left\{x \in c_{0}:\|x\|=1\right\}$. Then $S$ is a closed set in $\left(c_{0},\|\|\right)$. Denote by $e_{n}$ the unit vector in $c_{0}$ which has 1 at the $n$th coordinate and has 0 elsewhere. Then every $e_{n} \in S$ and 
$e_{n} \stackrel{n}{\rightarrow} 0$ in $\left(c_{0}, \sigma\left(c_{0}, l^{1}\right)\right)$. But $0 \notin S$ and hence $S$ is not weakly sequentially closed.

Example 2.2. We have $\left(l^{1},\|\|_{1}\right)^{*}=l^{\infty}$. Since every weakly convergent sequence in $l^{1}$ is norm convergent (see [12, Prob. 8-1-8]), each norm closed set in $\left(l^{1},\|\|\right)$ is weakly sequentially closed. Set $A=\left\{y \in l^{1}:\|y\|_{1} \geq 1\right\}$. Then $A$ is norm closed and hence weakly sequentially closed. But $A$ is not weakly closed. In fact, $A$ is weakly dense in $\left(l^{1},\|\|_{1}\right)$ (see [12, Prob. 8-1-6]).

By the definitions we have the following obvious implications. For closed bounded convex sets in a Fréchet space, the drop property implies the weak drop property, and the weak drop property implies the quasi-weak drop property. Since in a Fréchet space a weakly sequentially closed set need not be weakly closed (see Example 2.2), one may suppose that the quasi-weak drop property should be strictly weaker than the weak drop property. However we shall show that for closed bounded convex sets in a Fréchet space, the quasi-weak drop property is actually equivalent to weak compactness and hence it is equivalent to the weak drop property.

TheORem 2.2. Let $(X, d)$ be a Fréchet space and $B$ a closed bounded convex subset of $X$. Then the following statements are equivalent:

(i) $B$ is weakly compact.

(ii) $B$ has the weak drop property.

(iii) Every stream in $X \backslash B$ has a weakly convergent subsequence.

(iv) $B$ is weakly countably compact, i.e., every sequence in $B$ has a weak cluster point in $B$.

(v) $B$ has the quasi-weak drop property.

(vi) Eevery stream in $X \backslash B$ has a weak cluster point.

Proof. We shall prove the following two loops of implications: (i) $\Rightarrow($ ii $) \Rightarrow$ (iii) $\Rightarrow$ (vi) $\Rightarrow$ (i) and (i) $\Rightarrow$ (iv) $\Rightarrow(\mathrm{v}) \Leftrightarrow($ vi $) \Rightarrow$ (i).

(i) $\Rightarrow$ (ii). For any weakly sequentially closed set $A$ disjoint from $B$, we show that $A-B$ is weakly sequentially closed. Let $\left(a_{n}-b_{n}\right)_{n \in \mathbb{N}}$ be weakly convergent to $z_{0}$, where $a_{n} \in A$ and $b_{n} \in B$. Since $B$ is a weakly compact subset of the Fréchet space $(X, d), B$ is also weakly sequentially compact (see, for example, [6, I, p. 311]). Hence there exists a subsequence $\left(b_{n_{k}}\right)_{k \in \mathbb{N}}$ of $\left(b_{n}\right)_{n \in \mathbb{N}}$ which is weakly convergent to $b_{0} \in B$. Thus $\left(a_{n_{k}}-b_{n_{k}}\right)_{k \in \mathbb{N}}$ is weakly convergent to $z_{0}$ and $\left(b_{n_{k}}\right)_{k \in \mathbb{N}}$ is weakly convergent to $b_{0} \in B$. From this, $\left(a_{n_{k}}\right)_{k \in \mathbb{N}}$ is weakly convergent to $z_{0}+b_{0}$. As $A$ is weakly sequentially closed, we conclude that $z_{0}+b_{0} \in A$ and $z_{0}=\left(z_{0}+b_{0}\right)-b_{0} \in A-B$. That is, $A-B$ is weakly sequentially closed and hence $A-B$ is closed in $(X, d)$. Thus $A-B=\operatorname{cl}(A-B)$. Since $A$ is disjoint from $B$, we see that $0 \notin A-B=\operatorname{cl}(A-B)$, which leads to $d(A, B)>0$. Thus for any $z \in A$, 
$d(D(z, B) \cap A, B) \geq d(A, B)>0$. By Lemma 2.2, there exists an $x_{0} \in A$ such that $D\left(x_{0}, B\right) \cap A=\left\{x_{0}\right\}$. That is to say, $B$ has the weak drop property.

(ii) $\Rightarrow$ (iii). The proof is similar to the first half of the proof of $[2$, Theorem 2]. Suppose that there exists a stream $\left\{x_{n}\right\}$ in $X \backslash B$ without a weakly convergent subsequence. Then $A:=\left\{x_{n}: n \in \mathbb{N}\right\}$ is weakly sequentially closed. Now $x_{n+1} \in D\left(x_{n}, B\right)$ for all $n \in \mathbb{N}$, hence there is no $n \in \mathbb{N}$ such that $D\left(x_{n}, B\right) \cap A=\left\{x_{n}\right\}$. That is, $B$ does not have the weak drop property.

(iii) $\Rightarrow$ (vi) and (i) $\Rightarrow$ (iv) are obvious.

(iv) $\Rightarrow(\mathrm{v})$. Since $B$ is weakly countably compact, we can easily prove that for any weakly closed set $A$ disjoint from $B, A-B$ is weakly sequentially closed. Thus $A-B$ is closed in $(X, d)$ and $0 \notin A-B=\operatorname{cl}(A-B)$. Similarly to the proof of (i) $\Rightarrow\left(\right.$ ii), by Lemma 2.2 we deduce that there exists an $x_{0} \in A$ such that $D\left(x_{0}, B\right) \cap A=\left\{x_{0}\right\}$, which means that $B$ has the quasi-weak drop property.

$(\mathrm{v}) \Leftrightarrow(\mathrm{vi})$. See Theorem 2.1.

$(v i) \Rightarrow(\mathrm{i})$. The proof is an easy modification of the proof of Proposition 4.4.7 in [8]. Fix $f \in X^{*} \backslash\{0\}$. Clearly $M:=\sup \{f(x): x \in B\}<\infty$. We may assume that $0 \in B$ and $M>0$. Choose $x_{1} \notin B$ such that

$$
f\left(x_{1}\right)>\frac{5}{4} M
$$

and choose $y_{n} \in B$ such that

$$
f\left(y_{n}\right)>\left(1-\frac{1}{2 \cdot 4^{n}}\right) M, \quad n \in \mathbb{N} .
$$

We define a sequence $\left\{x_{n}\right\}$ recursively by $x_{n+1}:=\left(x_{n}+y_{n}\right) / 2$. By induction we can verify that for each $n$,

$$
f\left(x_{n}\right)>\frac{4^{n}+1}{4^{n}} M>M .
$$

By (vi), the stream $\left\{x_{n}\right\}$ has a weak cluster point $x_{0}$. Thus $f\left(x_{0}\right)$ is a cluster point of the scalar sequence $\left\{f\left(x_{n}\right)\right\}$. Since $f\left(x_{n}\right)>M$ for all $n \in \mathbb{N}$, we have $f\left(x_{0}\right) \geq M$. On the other hand, by induction we easily obtain

$$
\begin{aligned}
x_{n+1} & =\frac{1}{2^{n}} x_{1}+\frac{1}{2^{n}} y_{1}+\frac{1}{2^{n-1}} y_{2}+\ldots+\frac{1}{2} y_{n} \\
& =\frac{1}{2^{n}}\left(x_{1}-y_{1}\right)+\left(\frac{1}{2^{n-1}} y_{1}+\frac{1}{2^{n-1}} y_{2}+\ldots+\frac{1}{2} y_{n}\right) \\
& \in \frac{1}{2^{n}}\left(x_{1}-y_{1}\right)+\operatorname{conv}\left\{y_{1}, \ldots, y_{n}\right\} \\
& \subset \frac{1}{2^{n}}\left(x_{1}-y_{1}\right)+B .
\end{aligned}
$$


From this,

$$
x_{n+1}-\frac{1}{2^{n}}\left(x_{1}-y_{1}\right) \in B .
$$

It is easy to see that $x_{0}$ is also a weak cluster point of $\left\{x_{n+1}-\left(x_{1}-y_{1}\right) / 2^{n}\right\}$. Since the closed convex set $B$ is weakly closed, we have $x_{0} \in B$. Thus $f\left(x_{0}\right) \leq M$ and hence $f\left(x_{0}\right)=M$. That is, $f$ attains its supremum on $B$ so from James' characterization for weakly compact sets [5], $B$ is weakly compact.

By Theorem 2.2 and a characterisation of reflexive spaces (see, for example, $[4, \S 8]$ or $[6, \mathrm{I}, \mathrm{p} .303]$ ), we immediately obtain the following.

Corollary 2.1. Let $(X, d)$ be a Fréchet space (in particular a Banach space). Then the following statements are equivalent:

(i) $(X, d)$ is reflexive.

(ii) Every closed bounded convex set in $(X, d)$ has the weak drop property.

(iii) Every closed bounded convex set in $(X, d)$ has the quasi-weak drop property.

Moreover we may consider extending Theorem 2.2 and Corollary 2.1 to general locally convex spaces. But since there are various kinds of completeness in locally convex spaces, the situation becomes more complicated. be:

Recall that (see, for example, $[6])$ a locally convex space $(X, \tau)$ is said to

(a) complete if every Cauchy net in $(X, \tau)$ is convergent,

(b) quasi-complete if every bounded Cauchy net in $(X, \tau)$ is convergent,

(c) sequentially complete if every Cauchy sequence in $(X, \tau)$ is convergent,

(d) locally complete if every bounded set in $(X, \tau)$ is contained in a Banach disk.

We have the following obvious implications: complete $\Rightarrow$ quasi-complete $\Rightarrow$ sequentially complete $\Rightarrow$ locally complete.

But none of the converses of the above implications is true. For further details, we refer to [10] and [9, Chapter 5].

To end this paper we raise the following problems:

Problems. Let $B$ be a closed bounded convex set in a locally complete (respectively, sequentially complete, or quasi-complete) locally convex space $(X, \tau)$. Is the statement " $B$ has the quasi-weak drop property" equivalent to " $B$ is weakly compact"? For a locally complete (respectively, sequentially complete, or quasi-complete) locally convex space $(X, \tau)$, is the statement "every closed bounded convex subset of $X$ has the quasi-weak drop property" equivalent to " $(X, \tau)$ is semi-reflexive"? 
The author would like to thank the referee for very constructive suggestions on the first draft of this paper.

\section{References}

[1] J. Daneš, A geometric theorem useful in nonlinear functional analysis, Boll. Un. Mat. Ital. 6 (1972), 369-372.

[2] J. R. Giles and D. N. Kutzarova, Characterisation of drop and weak drop properties for closed bounded convex sets, Bull. Austral. Math. Soc. 43 (1991), 377-385.

[3] J. R. Giles, B. Sims and A. C. Yorke, On the drop and weak drop properties for a Banach space, Bull. Austral. Math. Soc. 41 (1990), 503-507.

[4] J. Horváth, Topological Vector Spaces and Distributions, Vol. 1, Addison-Wesley, Reading, MA, 1966.

[5] R. C. James, Weakly compact sets, Trans. Amer. Math. Soc. 113 (1964), 129-140.

[6] G. Köthe, Topological Vector Spaces I, II, Springer, Berlin, 1969, 1979.

[7] D. N. Kutzarova, On the drop property of convex sets in Banach spaces, in: Constructive Theory of Functions 1987 (Sofia, 1988), 283-287.

[8] D. Pallaschke and S. Rolewicz, Foundations of Mathematical Optimization, Math. Appl. 388, Kluwer, Dordrecht, 1997.

[9] P. Pérez Carreras and J. Bonet, Barrelled Locally Convex Spaces, North-Holland Math. Stud. Vol. 131, Amsterdam, 1987.

[10] J. H. Qiu, Local completeness and dual local quasi-completeness, Proc. Amer. Math. Soc. 129 (2001), 1419-1425.

[11] S. Rolewicz, On drop property, Studia Math. 85 (1987), 27-35.

[12] A. Wilansky, Modern Methods in Topological Vector Spaces, McGraw-Hill, New York, 1978.

Department of Mathematics

Suzhou University

Suzhou, Jiangsu 215006

People's Republic of China

E-mail: qjhsd@sina.com.cn

Received September 26, 2001

Revised version December 17, 2001 